\title{
Integration of Mental Health into Primary Health Care in a rural district in Uganda
}

\author{
J Ssebunnya', F Kigozi², D Kizza², S Ndyanabangi³, MHaPP Research Programme Consortium ${ }^{4}$ \\ 'Department of Mental Health and Community Psychology, Makere University, Kampala, Uganda \\ Butabika National Referral and Teaching Mental Hospital, Kampala, Uganda \\ ${ }_{3}$ Mental Health Division, Ministry of Health, Kampala, Uganda \\ ${ }^{4}$ The Mental Health and Poverty Project (MHaPP) is a Research Programme Consortium (RPC) funded by the UK Department for \\ International Development (DfID)(RPC HD6 2005- 2010) for the benefit of developing countries. The views expressed are not necessarily \\ those of DfID. RPC members include Alan J. Flisher (Director) and Crick Lund (Co-ordinator) (University of Cape Town, Republic of \\ South Africa (RSA)); Therese Agossou, Natalie Drew, Edwige Faydi and Michelle Funk (World Health Organization); Arvin Bhana (Human \\ Sciences Research Council, RSA); Victor Doku (Kintampo Health Research Centre, Ghana); Andrew Green and Mayeh Omar (University \\ of Leeds, UK); Fred Kigozi (Butabika Hospital, Uganda); Martin Knapp (University of London, UK); John Mayeya (Ministry of Health, \\ Zambia); Eva N Mulutsi (Department of Health, RSA); Sheila Zaramba Ndyanabangi (Ministry of Health, Uganda); Angela Ofori-Atta \\ (University of Ghana); Akwasi Osei (Ghana Health Service); and Inge Petersen (University of KwaZulu-Natal, RSA).
}

\begin{abstract}
Objective: Mental health has been identified as a major priority in the Ugandan Health Sector Strategic Plan. Efforts are currently underway to integrate mental health services into the Primary Health Care system. In this study, we report aspects of the integration of mental health into primary health care in one rural district in Uganda. Method: Qualitative methods were used for data collection. Semi-structured interviews and focus group discussions were conducted with various stakeholders within the ministry of health as well as line ministries. Data analysis was done using Nvivo 7, specifically adopting framework analysis approach. Results: Attempts to offer organized mental health services were found to be present in only a few health facilities. The district had only a single mental health nurse, and very few General Health Workers adequately equipped with the knowledge and skills to provide mental health services. The vertical referral system was not being followed as planned and there was no evidence of any organized community interventions for those with mental disorders. The mental health nurse and a few PHC nurses however expressed interest and commitment to providing services for persons with mental illness, despite the challenges. Conclusion: Although mental health is expected to be integrated into primary health care, mental health services in this district have not yet achieved the expected level of integration. This implies that this important policy requirement has not yet been effectively realized in this rural district, which could be the case in many other districts of a similar status. There is thus a need to direct more efforts towards realization of this important policy requirement.
\end{abstract}

Key words: Mental health; Primary Health Care; Integration; Uganda

Received: 07-11-2008

Accepted: 17-02-2009

\section{Introduction}

Over the last two decades, several initiatives have been undertaken to improve psychiatric services in low-income rural areas in developing countries. These have included the formulation of national mental health priorities and programs, or undertaking reforms of the existing mental health policies and strategies in line

\section{Correspondence:}

Joshua Ssebunnya

Department of Mental Health and Community Psychology,

Makerere University, Makerere, Uganda

email: joy95h@yahoo.co.uk with the overall health reforms. These reforms are intended to improve access and equity for the community to mental health services, through integration of mental health into primary health care. ${ }^{1,2}$

The rationale for the strategy of integrating mental health into primary health care is based on improved access to care, reduction in stigma, improved social integration, and improved human resources for mental health. ${ }^{3}$ However, although integration of mental health care into the existing primary health care system has been widely advocated and adopted as one of WHO's most fundamental health care recommendations, it has not been subjected to evaluation. Such evaluation would provide evidence 
needed to stimulate investment. There have been challenges as regards the implementation and practical realities on the ground. These include: insufficiency of resources, minimal funds being allocated to mental health due to the apparent low priority and false impression that mental health is not important, limited public enlightenment about mental health, insufficient training and supervision of Primary Health Care staff to detect and treat mental disorders, and their reluctance to work with people having mental disorders. ${ }^{1,3-6}$

Primary Health Care (PHC) implies providing 'essential health care' which is universally accessible to individuals and families in the community as near as possible to where people live and work. It also refers to care which is based on the needs of the population. Services should be decentralized, with active participation of the communities and family members., 1,8 Providing mental health services in primary health care involves diagnosing and treating people with mental disorders; putting in place strategies to promote and prevent mental disorders; and ensuring that primary heath care workers are able to apply key psychosocial and behavioral science skills, such as interviewing, counselling and interpersonal skills, in their day to day work. ${ }^{3}$

In Uganda, primary health care provided by general health workers, some of whom are not medically qualified forms the bedrock of the health care system. For some years, plans have been in place to integrate mental health care into the primary health care system as a way of improving access to mental health services for the Ugandan population. Uganda's health policy has decentralized the provision of health services to districts. Cluster 4 of the framework for the delivery of the Minimum Health Care Package constitutes the non-communicable diseases, of which mental health and substance abuse are key elements. ${ }^{9}$

This paper describes mental health services in Mayuge, a rural district in Uganda from the perspective of mental health care as an integrated component of primary health care. The paper comprises an output of the Mental Health and Poverty Research Project, which had as one of its objectives to carry out a situation analysis of the mental health situation in Uganda. The project is a 5year Research Programme Consortium which aims at developing the scientific knowledge base on how effective mental health policy development and implementation can assist in breaking the negative cycle of poverty and mental ill-health in low and middle income countries. ${ }^{10}$ Mayuge is one of the districts where the study was carried out, with a purpose of examining the effectiveness of the integration of mental health into primary health care in the rural health care system in Uganda.

\section{Method}

The study district (Mayuge) is located in the eastern region of the country, $120 \mathrm{~km}$ from the capital city, and was created in 2000 from another existing district. The district has a size of 4,672.22 $\mathrm{Km}^{2}$ and a population of 326,567 (2006 National Census). ${ }^{11,12}$ The district's health service delivery consists of 16 government dispensaries, 3 health centres at county level and 2 health centres at sub-district level. There are also 5 private dispensaries, 2 private clinics and 1 private hospital; formerly a leprosy treatment centre. ${ }^{12}$ This district was selected because it is typical of many rural districts in Uganda. It is also part of the worldwide INDEPTH network, and a Demographic Surveillance Site which continuously provides demographic and health information.

Qualitative methods were used to collect data for this particular objective of the study. Semi-structured interviews and focus group discussions were conducted for this purpose. Selection of the participants in the study was purposeful, specifically targeting those key informants who were believed to be resourceful. The participants included health care managers, primary health care workers, health facility managers social workers, teachers as well as other key informants from relevant sectors such as education, social welfare, law and justice. This paper is thus based on data collected from 9 in-depth interviews and 4 focus group discussions, each consisting of 5-7 participants. In-depth interviews are an effective qualitative method useful for learning about the perspectives of individuals as opposed to group norms of a community. ${ }^{13}$ The interviews and focus group discussions were conducted in English, audio-recorded and transcribed verbatim. The transcriptions were then coded, and analysis done using Nvivo 7 qualitative data analysis software. Thematic analysis was done, specifically adopting a framework analysis approach ${ }^{14}$, as this was a multi-centre study. By this approach, certain themes and sub-themes were agreed upon by the investigators at all the research sites, based on the objectives of the study. Thus, a single framework for analysis was developed, and specific views emerging from the interviews were added into the framework in the process. The transcriptions were thus coded on the basis of a pre-determined coding frame.

\section{Results}

The major findings concerning integration of mental health care are organized into three themes: the mental health system and financing, mental health staffing and training, and management of patients.

\section{The mental health system and financing}

Mental health services were found to be relatively new in this district, with only a few health facilities making attempts to attend to patients with mental health problems. The district health management had no mental health plan and no well organized mental health programs running. With no specific mental health budget, mental health was said to draw from the general PHC budget as an integrated component, with no predetermined budgetary allocations:
'... if you look through my work plan here, you will not easily tease a bit of mental health; but it is integrated within. I mean we have the health sector strategic plan of which we are looking at how we really spell out which direction we should be taking for mental health. So, that is basically it. We have it in plan, but implementation may be rather different"' (SSI, District Services Manager)

It was also noted that there was no significant intersectoral collaboration for mental health in this district, neither were there any immediate plans to establish this collaboration. Some of the participants from other non-health sectors that have links with mental health (such as law and justice, social welfare) were noted to be uninformed about mental health. They envisaged no need of some basic training in mental health related issues, and believed that mental health is completely outside their jurisdiction. The district political leadership was reported to be characterized by lots of internal conflicts and administrative problems especially attributable to political squabbles; something that was believed to be typical of most new districts. These problems were said to grossly affect service delivery in the district. 


\section{Mental health staffing and training}

The staffing levels for mental health in the district were noted to be far below the optimal. There was only one mental health nurse in the district, who had just been recruited at one of the health centres. This mental health nurse however reported often being assigned other duties as a general nurse. The general hospital had no provision for a mental health worker, as staffing was reported to be dependent on the key priority areas to them; and yet mental health was taken to be a non-priority area in the district. The hospital manager affirmed that general nurses take precedence, and believed that the available medical officers would be in position to attend to patients. He was quoted saying:

\begin{abstract}
"... we don't have mental health staff in the hospital. Mental health has not been one of the immediate priority areas... we look at immediate priorities. I can't bring in a psychiatric nurse if don't have the general nurses to take care of general patients"
\end{abstract}

The only mental health nurse for the district reported receiving no support supervision from senior mental health professionals from the Regional Hospital, and thus expressed fear of degeneration of the knowledge with time. Although the health managers believed that most health workers had had a component of mental health in their curricula during the training, most PHC nurses admitted never having received any training in mental health. It also emerged that most PHC workers with some basic training in mental health do not regard managing the mentally ill as their primary role, other than identification and referral.

\section{Management of patients}

It emerged that efforts to provide some kind of mental health care in the rural communities are still partly constrained by the belief system. Most participants affirmed that the cultural explanatory model for mental illness still prevails, and most patients and carers are reluctant to seek help at the health facilities. Furthermore, patients and carers were reported to have less confidence in the mental health services at the health facilities. Instead, they seek help from traditional healers most of the time. The health workers at the general hospital admitted being able to identify and attend to only the overtly mentally ill patients. It was noted that many patients with less obvious mental illnesses or those with mental illnesses presenting primarily as physical symptoms are usually not easily identified at the facilities, and therefore their mental health problems are often left unattended to.

Only a few mental health drugs, particularly anti-epileptics were reported to be available at some of the health facilities, with irregular supply most of the time. The few Primary Health Care doctors available were noted to have minimal interest in mental health. These doctors and other PHC workers believed that their role in mental health care is identification and referral of patients, with no managerial roles. However, although an upward referral system exists, it is never adhered to, as patients seek help where they expect the services. Most patients bypass the available levels of care, seeking help directly at the Regional Referral hospital or further away at the National Referral Mental Hospital in the capital city.

A particularly striking finding was that even in the absence of mental health personnel, PHC workers trained in mental health, and an explicit mental health plan in place the health managers maintained that mental health care is part of the service delivery as an integrated component; a point echoed by one of the context informants:
"....As of now, I wouldn't say that its activities are really teased out. They are completely integrated into our work of PHC (primary health care). Well as a district we don't have a particular unit that handles mental health as such and I would say we don't have many specialists. But by and large, it doesn't mean that mental health is not completely taken care of...in one way or another we may not be so specialized but we can do some thing about mental health" (SSI, Health Services Manager)

He added:

\section{"....indirect... indirectly integrated within the network of treatment and care that we have. We may not be particularly targeting mental illness but we are treating a person holistically".}

The health managers further believed that all health workers receive some training in mental health during the pre-service training and are therefore able to attend to the mental health needs of patients, although this was not clearly demonstrated in practice. However, the mental health nurse and a few of the PHC nurses expressed commitment and interest in mental health care despite the challenges within the system.

\section{Discussion}

The findings indicate a discrepancy between the assumptions by health managers and the reality on ground, as regards the integration of mental health. It is clear that some health managers believe the integration of mental health into PHC occurs automatically even in absence of deliberate efforts to operationalize the policy requirement. It should be noted that integration of mental health into Primary Health Care is a national policy with specific targets such as increasing community access to mental health services by at least $50 \%{ }^{9}$ which calls for well planned efforts, to be realized.

It is important that health workers understand what integration entails and how it can be achieved. A number of writers have attempted to describe how mental health services and primary health care can be linked; and some of these models are applicable internationally. In the US, three models have been described: (1) the primary health care practitioners receive limited specialist mental health training; (2) specialist mental health practitioners consult with primary care practitioners on a case-by-case basis; and (3) mental health practitioners serve as part of the primary care team. ${ }^{7}$ The findings indicated that none of the above three models is at least moderately applied in the district.

The Alma-Ata declaration (1978) acknowledges that realization of health requires action of many other sectors in addition to the health sector. ${ }^{8}$ This district's health department was noted to have no links with other departments in favour of mental health. It should be noted that an intersectoral approach and collaboration would enhance success of the primary health care approach mainly through collaboration with non-health sectors such as education, social welfare, justice and labour at policy, planning and service levels. 
Integration of mental health into primary health care requires investment in the training of the staff to detect and treat common mental disorders. General health staff must have the knowledge, skills and motivation to treat and manage patients suffering from mental disorders as much as possible within their competencies. ${ }^{3}$ A mental health component should be included in the educational curriculum of all social and health workers and ongoing training and support supervision should always be provided on site. In respect to this district, the presence of just a single mental health nurse for the entire district indicated a miserably low staffing level that is not in line with the recommendation of the staffing norms. The local government staffing norms for district health services allow for a Nursing Officer (psychiatry) and an enrolled psychiatric nurse at a Health sub-District. At the District General Hospital level, provision is made for a Nursing Officer (psychiatry), 2 Enrolled Psychiatric Nurses and a Psychiatric Clinical Officer. 9,15 It was further unclear as to how many of the available PHC workers had received some training in mental health.

The general hospital manager was cognizant of the fact that mental health nurses can do general nursing as well, which would perhaps make them be of an advantage as compared to the general nurses. He however assumed that the mental health nurses would prefer doing mental health related work only and hence the reluctance to give them strong consideration. This apparently reflects a prejudiced attitude towards mental health. Although the hospital manager reported that medical officers attend to patients with mental illness, the latter declined to take part in the study, stating that they are not mental health professionals. This casts doubt as regards their interest and readiness to attend to patients with mental health problems.

The findings indicated that the Primary Health Care workers believed their role in mental health care to be that of identification and referral of patients. They tend to be uncomfortable dealing with mental disorders and may also question their role in managing mental disorders. Therefore, in addition to imparting skills, training also needs to address the overall attitude of primary health care workers to embrace persons with mental disorders. This calls for regular in-service training of all general health workers, which was lacking in the district. This also highlights the need for use and strengthening of existing networks of services to provide mental health services. ${ }^{3}$ With health managers who trained at a time when mental health received less consideration and with no organized Continuing Medical Education in the area of mental health, it became clear that the health workers are illequipped to handle patients with mental illnesses and that effective mental health care still remains a challenge.

Adequate supervision of primary care staff is another key issue which has to be addressed if integration is to succeed. Mental health professionals from the centre should be available regularly in the primary care settings to monitor and provide technical support supervision at all levels for the staff. This was noted to be lacking, which further casts doubt on the effectiveness of the integration process. Thus, the need for further training of the $\mathrm{PHC}$ workers in mental health and Continuous Medical Education (CME) in mental health was obvious, in view of the inadequate training and supervision.

While it is clear that basic psychotropic medicines must be available at primary and secondary care levels in an integrated health care system, it was noted that the psychotropic medicines are seldom available at the health facilities in the district. Being in a decentralized system, this illustrates the need for the district health management to ensure that sufficient funds are allocated to purchase the basic essential psychotropic medicines and also make sure that they are always available in primary care settings, in accordance with the policy adopted.

\section{Conclusion}

From the study findings, it was clear that although mental health is said to be integrated into Primary Health Care, mental health services in this district are still far from the expected level of integration. Furthermore, there is high suspicion that the situation is not much different in many other newly created and resource limited rural districts. Such challenges are likely to be the case in many settings where integration of mental health into Primary Health Care has been taken on, but with no critical follow up and evaluation of the integration process. This implies that this important policy requirement of integration has not yet been fully realized by the health system in the rural settings, such that access by the community to mental health services is still inadequate. This therefore calls for a deliberate strategy by the concerned health department(s) to scale up activities for effective integration of mental health into Primary Health Care in the rural settings, so as to enable the entire population access quality mental health care.

\section{References}

1. Kigozi F. Integrating Mental Health into Primary Health Care - Uganda's experience. South African Psychiatry Review 2007;10(1):17-9.

2. Sherer R. Mental Health Care in the Developing World. Psychiatric Times 2002;19(1):1-6.

3. WHO. Integrating Mental Health into Primary Health Care. Geneva, World Health Organization, 2007.

4. Shah A, Jenkins R. Mental health economic studies from developing countries reviewed in the context of those from developed countries. Acta Psychiatrica Scandinavica 1999;100:1-18.

5. WHO. World Health Report 2001, Mental Health: new understanding, new hope. World Health Organization, Geneva.; 2001.

6. Knapp M, Funk M, Curran C, Prince M, Grigg M, McDaid D. Economic Barriers to Better Mental Health Practice and Policy. Journal of Health Policy and Planning 2006;21 (3):157-70.

7. Gask L. Overt and covert barriers to the integration of primary and specialist mental health care. Social Science \& Medicine 2005; (61):178594

8. WHO. Declaration of Alma-Ata. International Conference on Primary Health Care. Geneva, 1978.

9. Ministry of Health. Health Sector Strategic Plan II. Ministry of Health, Kampala-Uganda, 2005.

10. Flisher AJ, Lund C, Funk M, Banda M, Bhana A, Doku V et al. Mental health policy development and implementation in four African countries. Journal of Health Psychology 2007;12:505-16.

11. Uganda Bureau of Statistics. State of Uganda Population Report, 2006.

12. Mayuge District Council. About Mayuge. Www.mayuge.go.ug

13. MackN, Woodsong C, MacQueen KM, Guest G, Namey. Qualitative Research Methods. A Data Collector's Field Guide. Family Health International, USA. 2005.

14. Ritchie J, Spencer L. Qualitative data analysis for applied policy research. In Bryman \& Burgess (Eds.). Analysing qualitative data 1994;173-94.

15. Health Service Commission. Recruitment Guidelines for Health Professionals in Districts and Urban Authorities. Ministry of Health, Kampala, 2005. 